Article

\title{
The rising role of age stratification in sleep and CPAP therapy adherence in elderly population
}

\author{
Silvia Gavliakova ${ }^{1,2}$, Vladimira Bellova ${ }^{2}$
}

How to cite this paper: Gavliakova, $\mathrm{S}$. The Rising role of age stratification in sleep and CPAP therapy adherence in elderly population. Open Journal of Medical Sciences. DOI: 10.31586/ojms.2021.010103. Retrieved from https://www.scipublications.com/journal/index.php/ojms/article/view/21

Received: May 12, 2021

Accepted: June 20, 2021

Published: June 21, 2021

Copyright: (c) 2021 by the authors. Submitted for possible open access publication under the terms and conditions of the Creative Commons Attribution (CC BY) license (http://creativecommons.org/licenses /by/4.0/).
1(Affiliation 1): Jessenius Faculty of Medicine in Martin, Comenius University in Bratislava, Martin, Slovakia 2(Affiliation 2): Clinic of Pneumology and Phthisiology, University Hospital Martin, Martin, Slovakia

\begin{abstract}
Proper and effective management of obstructive sleep apnea (OSA) in elderly patients represents an important yet under-researched therapeutic target. Therefore, the main purpose of our research was to employ age stratification to analyse sleep quality and sleep fragmentation, the daily sleepiness, OSA severity and CPAP compliance in elderly. Conforming to the inclusion criteria of minimum 70 years of age at the time of polysomnography and CPAP titration night a total number of 162 elderly patients was included, median age 73,00 \pm 4 (MED $\pm \mathrm{IQR})$. The comparison group

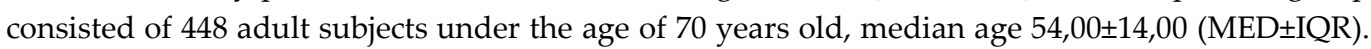
Sleep fragmentation in elderly was promoted to suboptimal CPAP adherence, as the probability of CPAP use $\geq 4$ hours per day was less than $20 \%$. Overall CPAP compliance hours in the elderly group achieved 3,991 $\pm 3,804$ hours/day with bigger variation in between the subjects versus 5,547 $\pm 3,465$ hours/day for the controls, (MED $\pm I Q R, p<0,001)$. Despite naturally occurring sleep fragmentation worsened by OSA, less than $30 \%$ of elderly patients would score $\geq 10$ points on Epworth sleepiness scale. The study emphasizes acute need for standardized age-specific diagnostic tools to address different perception of daily drowsiness and sleepiness by older people. Further, it underlines the importance of early recognition of possible comorbid OSA and insomnia and suggest paying more attention to "non-obese phenotype" in the subgroup of elderly patients with suspected OSA. Elderly patients might also need development of more flexible CPAP compliance criteria; however those must be standardized and objective.
\end{abstract}

Keywords: Senior; CPAP; COMISA; Obstructive sleep apnea; Sleepiness, Geriatric sleep

\section{Introduction}

Obstructive sleep apnea (OSA) is common in the population with prevalence increasing with age and continuous positive airway pressure (CPAP) therapy represents a golden standard in the treatment of obstructive sleep apnea syndrome. The prevalence of sleep disturbed breathing in older people in the general population can vary from $20 \%$ to $40 \%$ [1]. Data from large cohorts also demonstrate that the prevalence of sleep disturbed breathing disorders steadily increases with advancing age, with a plateau between 60 and 65 years of age [2]. Sleep disordered breathing and inadequate quality or quantity of sleep in the older age adds to sleep fragmentation and has adverse effects on cardio-cerebrovascular, cognitive and functional parameters [3]. Subjectively, elderly people describe their daily sleepiness and their health and fitness in general differently when compared to younger counterparts $[4,5]$.

Effective CPAP therapy undeniably improves overall quality of life, reduces fatigue and sleep-related symptoms, anxiety and depression indexes and some neurocognitive aspects in people with severe OSA [6]. However, studies in OSA treatment that include age stratification mainly focus on middle-aged and younger patient groups. Hence, the consensus of the International Geriatric Sleep Medicine Task Force, published in European Respiratory Journal declared lack of relevant research in the area of sleep disturbed 
breathing in elderly patients [7]. Addingly, the final report of the USA National Sleep Foundation's updated sleep duration recommendations from 2015 highlights that the ratio of daily sleepiness caused due to sleep disordered breathing in older people remains undefined [8].

The role CPAP in the treatment of elderly patients with OSA is not sufficiently defined, as there are many factors to take into consideration, eg. physiological increase in the number of sleep-related disorders with the passing of years, the lack of validated diagnostic and therapeutic tools for this age group or proper ways to diagnose and treat OSA in the elderly. Sleep related breathing disorders in seniors are commonly overlapped with sleep disorders such as insomnia, sleep fragmentation or circadian rhythm disturbances. However, even with advancing age, the CPAP therapy demonstrated benefits for OSA patients such as improvement of cognition and overall health status $[9,5]$.

As the general population ages, the elderly population is becoming more and more important, but on the other hand, sleep disorders and their management become more complicated by a number of concurrent comorbidities. Literature reviews name several factors with significant effect on sleep quality, micro- and macroarchitecture of sleep in the aging population (Table 1, Table 2).

Table 1. Most common causes of sleep disorders in geriatric patients [10, 11].

\begin{tabular}{|c|c|c|}
\hline \multirow[t]{2}{*}{$\begin{array}{l}\text { Neurological } \\
\text { conditions }\end{array}$} & $\begin{array}{c}\text { Parkinson's disease, Alzheimer's disease, } \\
\text { Amyotrophic lateral sclerosis }\end{array}$ & $\begin{array}{l}\text { Sleep fragmentation, lowered mo- } \\
\text { tor control = difficulty in changing } \\
\text { and maintaining convenient sleep } \\
\text { position }\end{array}$ \\
\hline & $\begin{array}{c}\text { REM sleep-behavior disorders, Restless legs } \\
\text { syndrome, Periodic limb movements in } \\
\text { sleep }\end{array}$ & \\
\hline \multirow[t]{2}{*}{ Psychiatric } & $\begin{array}{l}\text { Bipolar disorders, schizophrenia, posttrau- } \\
\text { matic stress disorder, anxiety disorders }\end{array}$ & $\begin{array}{l}\text { Difficulty initiating and/or main- } \\
\text { taining sleep }\end{array}$ \\
\hline & Dementia, Depression, Anxiety & Insomnias \\
\hline \multirow[t]{2}{*}{ Cardiac } & $\begin{array}{c}\text { Left ventricular failure with orthopnea and } \\
\text { paroxysmal nocturnal dyspnea }\end{array}$ & \\
\hline & Tachyarrhythmias & \\
\hline \multirow[t]{2}{*}{ Respiratory } & $\begin{array}{l}\text { Sleep disordered breathing (Cheyne-Stokes } \\
\text { breathing, Obstructive sleep apnea, Obesity } \\
\text { hypoventilation syndrome...) }\end{array}$ & \\
\hline & $\begin{array}{l}\text { Chronic obstructive pulmonary disease } \\
\text { (COPD), Asthma }\end{array}$ & $\begin{array}{l}\text { Nocturnal worsening of hypox- } \\
\text { emia, predominantly during REM } \\
\text { sleep }\end{array}$ \\
\hline Urinary & $\begin{array}{l}\text { Benign prostatic hypertrophy, Detrusor in- } \\
\text { stability }\end{array}$ & \\
\hline Chronic pain & $\begin{array}{l}\text { Vertebral algic syndromes, Osteoarthritis, } \\
\text { Metastatic diseases }\end{array}$ & \\
\hline $\begin{array}{l}\text { Circadian } \\
\text { rhythm dis- } \\
\text { turbances }\end{array}$ & Daytime napping, Jet-lag & \\
\hline Other & $\begin{array}{c}\text { Gastroesophageal reflux disease (GERD) } \\
\text { Pruritic skin conditions } \\
\text { Medication side-effects } \\
\end{array}$ & See Table 2. \\
\hline
\end{tabular}


Table 2. Older patients take an average of 5-9 daily medications, some of which can interfere with sleep and wakefulness [12, 13].

\begin{tabular}{|c|c|}
\hline $\begin{array}{l}\text { Sedative antidepressants and neuroleptics (amitriptyline, } \\
\text { chlorpromazine, clozapine) }\end{array}$ & $\begin{array}{l}\text { Impaired performance and day- } \\
\text { time drowsiness }\end{array}$ \\
\hline Sedative antidepressant amitriptyline & $\begin{array}{l}\text { Anticholinergic effects, possible } \\
\text { confusion }\end{array}$ \\
\hline Sedative hypnotics (chronic use) & $\begin{array}{c}\text { Drug withdrawal effects, daytime } \\
\text { drowsiness }\end{array}$ \\
\hline $\begin{array}{l}\text { Beta-blockers (lipophilic compounds - metoprolol, proprano- } \\
\text { lol) }\end{array}$ & $\begin{array}{c}\text { Difficulty falling asleep, increased } \\
\text { number of awakenings, vivid } \\
\text { dreams }\end{array}$ \\
\hline $\begin{array}{l}\text { Xanthines (theophylline, caffeine - in over the counter medi- } \\
\text { cations eg. analgesics, cold and allergy remedies, appetite } \\
\text { suppressants) }\end{array}$ & $\begin{array}{l}\text { Increase wakefulness, decrease to- } \\
\text { tal sleep duration and slow wave } \\
\text { sleep duration }\end{array}$ \\
\hline $\begin{array}{c}\text { Cold and allergy remedies (containing caffeine and stimula- } \\
\text { tory decongestants } \\
\text { (phenylephrine, pseudoephedrine, phe- } \\
\text { nylpropanolamine) }\end{array}$ & $\begin{array}{l}\text { Stimulatory effects, headaches, } \\
\text { dizziness, restlessness }\end{array}$ \\
\hline $\begin{array}{c}\text { Diet pills (containing phenylpropanolamine, ephedrine, } \\
\text { pseudoephedrine, caffeine) }\end{array}$ & Stimulatory effects \\
\hline Nicotine & $\begin{array}{l}\text { Stimulatory effects, difficulty fall- } \\
\text { ing asleep, decreased sleep dura- } \\
\text { tion }\end{array}$ \\
\hline
\end{tabular}

Taking all these facts into consideration, the main purpose and significance of our research is to bring more valuable data and insights to quality of sleep and treatment of OSA in vulnerable population of older adults. The study employs age stratification to evaluate sleep quality and fragmentation, the proportion of spontaneous vs. respiratory related arousals, daily sleepiness, OSA severity and CPAP compliance.

\section{Materials and Methods}

The study was designed as a single-centered retrospective observational analysis of a pool of patients who underwent in-lab sleep study for snoring and sleep apnea related complications. The subjects were divided into two groups according to their age at the time when the sleep study was performed. The criteria were a minimum 70 years of age for the senior group $(n=162)$ and 18 - 69 years for controls $(n=448)$. The research was conducted according to the principles of the Declaration of Helsinki. The below described procedure is a standard protocol for polysomnography in our University hospital, as the Laboratory of Sleep medicine is integral part of in-patient's clinic and the Sleep lab admission protocol is derived from a routine admission procedure at pulmonary clinic. The data are not a part of any other study or research project.

\subsection{First contact: Otorhinolaryngologic examination}

Patients were sent for standard ear-nose-throat (ENT) examination to assess the health of the upper airways and to determine and manage any possible pathology responsible for their snoring and sleep apnea related complications.

\subsection{Second contact: Physical examination by pulmonologist/somnologist.}

All the patients were enquired about their past ENT history, any former surgical interventions or earlier lesions and injuries in the area of upper airways. Neck habitus and the actual Mallampati score was noted. Comorbidities such as arterial hypertension, cardiovascular history in general, respiratory allergies, chronic rhinitis, asthma, COPD, dia- 
betes mellitus, thyroid dysfunction, depression or anxiety disorders etc. were not excluded from this observational study and our interest was focused on a typical group of patients who commonly visit sleep centers.

Subsequently, a certified somnologist/pulmonologist took the patient's detailed history of prescribed pharmacotherapy and any over-the-counter supplements. Patient's daily habits were assessed with a focus on their sleeping routine. Pack-year smoking history was noted, together with current and past occupational status in relevance to respiratory health. Body mass indexes were calculated, and patients were asked about how their body weight changed in the past 10-20 years. Every patient completed a validated sleep questionnaire including Epworth sleepiness scale assessment.

\subsection{Third contact: Sleep study}

Either in-lab polysomnography (PSG) or respiratory polygraphy was indicated. Unattended home respiratory polygraphy served as a screening test for sleep apnea in the case of ambiguous findings of sleep apnea symptoms during physical examination. Subjects with clearly negative findings from respiratory polygraphy (apnea/hypopnea index $\mathrm{AHI}<5$ events per hour of time in bed) and no other relevant comorbidities (asthma, COPD, arterial hypertension, etc.) were not invited for a polysomnography test and therefore those subjects were excluded from the study.

Routine lung function tests (spirometry/bodyplethysmography), electrocardiogram and blood pressure readings were performed in the morning before PSG study. Patients were instructed not to drink caffeinated or alcoholic drinks or smoke cigarettes before the PSG. Arterialized capillary blood gas analysis was used to estimate arterial gas content on the morning after PSG study as well as after the continuous positive airway pressure titration night, both performed directly upon wake-up.

Polysomnography was performed and scored by a certified sleep technologist and further evaluated by a pulmonologist with a valid examination from sleep medicine who also determined the next diagnostic steps.

\subsection{In-lab procedure}

Polysomnography - standard equipment according to current AASM guidelines (Philips Respironics Alice 6 LDx polysomnograph, Alice NightOne and Alice PDX for respiratory polygraphy, Sleepware G3 software interface) with minimum recording duration time 420 minutes. Obstructive Sleep Apnea syndrome was categorized as follows: $A b-$ sence of $\mathrm{OSA}=\mathrm{AHI}<5$. Mild OSA $=\mathrm{AHI} \geq 5$ and $<15$. Moderate $\mathrm{OSA}=\mathrm{AHI} \geq 15$ and $<$ 30. Severe OSA $=\mathrm{AHI} \geq 30$.

Automatic or manual titration of therapeutic positive airway pressure was performed on the subsequent night with Omnilab and DreamStation Auto-CPAP devices from Philips-Respironics and PrismaLab from Lowenstein Medizin. Philips-Respironics Encore, Sleepware G3 and Lowenstein Medizin PTS software was used to control the titration devices and analyze the effectiveness of the therapy. After titration, prescription and initiation of the CPAP therapy, patients were invited for the next consultation after 3 - 12 months, depending on other comorbidities and individual health status.

\subsection{Analysis}

Parameters analysed: physical characteristics (age, gender, body-mass index, Epworth sleepiness scale), polysomnography parameters (sleep onset, depth and continuity, arousals, sleep stages, respiratory disturbance and apnea-hypopnoea indexes, oxygen saturation, etc.), positive airway pressure therapy parameters (pressure settings, daily compliance and adherence, mask choice).

Statistical analysis was performed using statistical software and data analysis addon for Microsoft Excel - XLSTAT and GraphPad Prism. Methods used were descriptive statistics, Kruskal - Wallis analysis, Mann-Whitney U test. Regression analysis was used 
for estimating the relationships between variables. Confidence interval $95 \%$, results were considered significant with $\mathrm{p}$ values less than 0,05 .

Table 3. Basic sleep parameters and adult reference values [14].

\begin{tabular}{|c|c|}
\hline Sleep duration & $380-450 \mathrm{~min}$ \\
\hline Sleep latency & $\leq 30 \mathrm{~min}$ \\
\hline REM latency & $60-100 \mathrm{~min}$ \\
\hline Sleep efficiency & $>85 \%$ \\
\hline WASO - Wake after sleep onset & $1-5 \%$ \\
\hline NREM1 & $3-5 \%$ \\
\hline NREM2 & $45-55 \%$ \\
\hline REM sleep (Rapid eye movement sleep) & $10-20 \%$ \\
\hline Apnea / hypopnea index & $20-25 \%$ \\
\hline Periodic limb movement index & $\leq 15$ \\
\hline Epoch scored as wake & $>15 \mathrm{~s}$ \\
\hline Arousal duration & $3-5 \mathrm{~s}$ \\
\hline Sleep cycle - duration & $90-110 \mathrm{~min}$ \\
\hline Sleep cycles per night & $4 \mathrm{x}-6 \mathrm{x}$ \\
\hline
\end{tabular}

\section{Results}

\subsection{Examination group - elderly}

Conforming to the inclusion criteria of minimum 70 years of age at the time of polysomnography and CPAP titration night a total number of 162 elderly patients could be included. The median age was 73,00 \pm 4 (MED $\pm \mathrm{IQR}$ ). The group consisted of $62,791 \%$ male and $37,209 \%$ female subjects. The oldest female patient was 87 years old, diagnosed with obstructive sleep apnea/hypopnoea index AHI=33,145 events per hour of total sleep time - hTST. The patient was optimistic when initiating CPAP therapy and reported improved sleep quality. However, her compliance decreased in the following 6 months due to recurrent seasonal upper airway reinfections. The oldest male patient was 81 years old and diagnosed with obstructive sleep apnea/hypopnoea index AHI=66,549/hTST. The patient wished to withdraw CPAP after 2 years of therapy, reporting lowered sleep quality due to CPAP. The CPAP machine induced bad dreams and feelings of anxiety in this patient, he also felt uncomfortable when wearing a respiratory mask.

Average duration of CPAP therapy was 686 days for the seniors.

\subsection{Control group}

The comparison group was composed of 448 adult subjects under the age of 70 years old, who visited the clinic and who were subsequently indicated for in-lab sleep study. Their median age was $54,00 \pm 14,00$ (MED \pm IQR). The gender effect on OSA syndrome was more observable in this group as the majority of patients in the comparison group was male $(71,704 \%)$. There was a significant difference in body-mass index (BMI) between the elderly patients and the comparison group. However, median BMI values stayed within $30-35 \mathrm{~kg} / \mathrm{m} 2$ interval, which corresponds to the first degree of obesity. The age distribution of the whole dataset is shown in a histogram representation (Figure 1, Table 4).

Average duration of CPAP therapy was 818 days for the comparison group. 


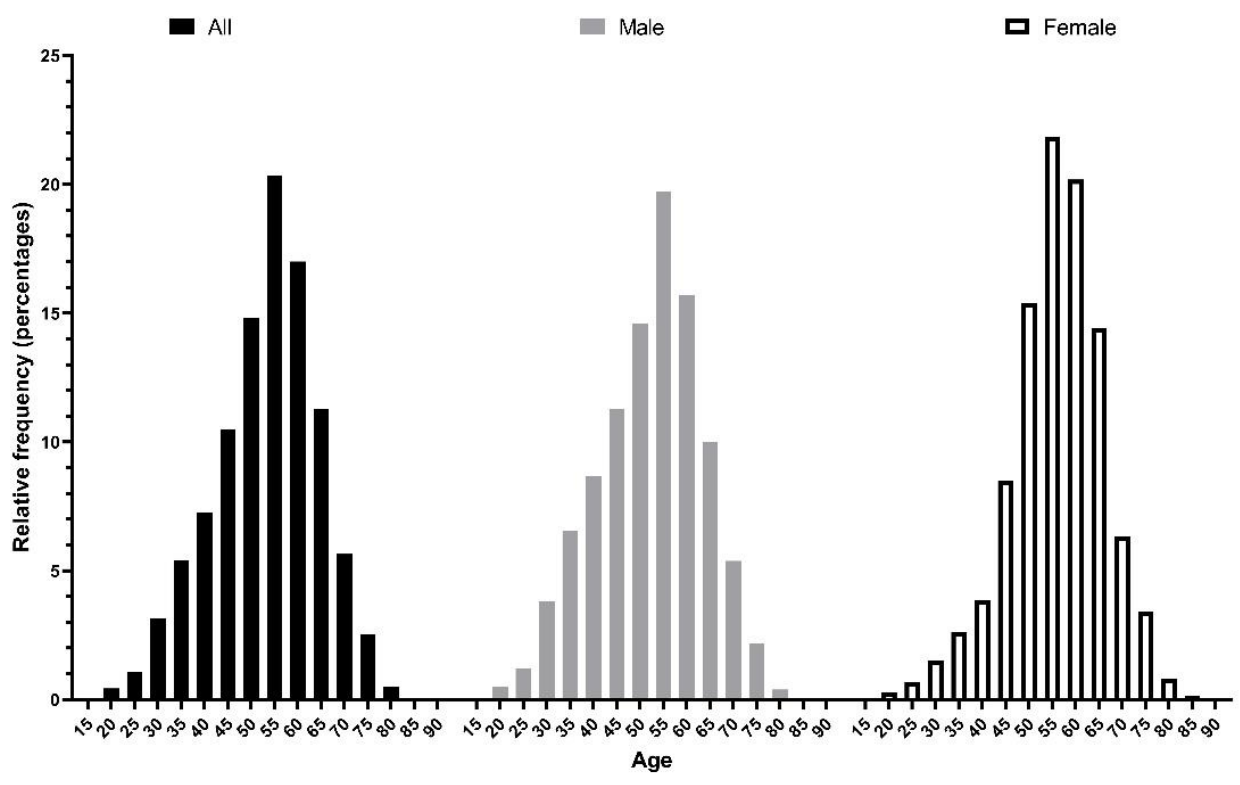

Figure 1. Histogram representation of age distribution of studied patients. The prevalence of OSA in female patients increases at menopause for reasons that include both weight gain and unclear hormonal mechanisms.

Table 4. Age distribution of studied patients. In general, women visit our sleep center older than male patients $\left({ }^{*} \mathrm{p}<0,0001\right)$.

\begin{tabular}{|c|c|c|c|}
\hline Age distribution & All & Male & Female \\
\hline Minimum & 19 & 19 & 20 \\
\hline 25\% Percentile & 46 & 44,5 & 50 \\
\hline Median & 54 & 53 & $56^{*}$ \\
\hline 75\% Percentile & 61 & 60 & 63 \\
\hline Maximum & 87 & 81 & 87 \\
\hline p-value male vs. female & \multicolumn{3}{|c|}{${ }^{*} \mathrm{p}<0,0001$} \\
\hline
\end{tabular}

\subsection{Polysomnography findings}

No significant differences were found in parameters such as proportion of NREM1 and REM sleep or REM sleep latency. Interestingly, while the onset of REM sleep or REM latency and also the percentual proportion of REM sleep was similar in both groups, the total duration of REM sleep and number of REM cycles was lower in older people (Table 5).

The sleep disturbed breathing and apnea/hypopnoea episodes have undoubtedly influenced the quality of sleep and the polysomnography parameters. But the data also clearly document, that sleep fragmentation, reduced sleep quality and the difficulties of sleep maintenance in elderly patients are a part of aging process only partially influenced by obstructive sleep apnea syndrome as there were no significant differences in apnea/hypopnoea and desaturation indexes between the groups (Table 5). Both groups had similar AHI and desaturation indexes in REM and non-REM sleep (Table 5, Figure 2, panels A, B, Figure 3). 
A

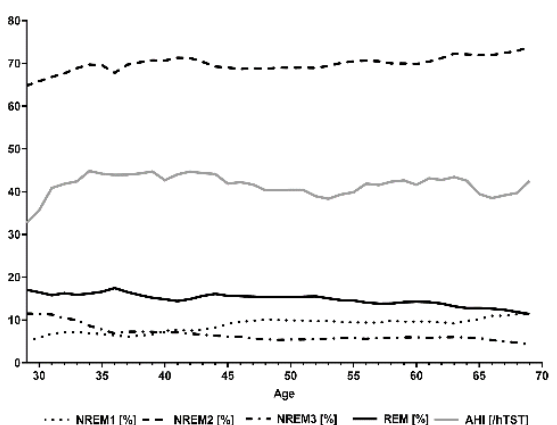

C

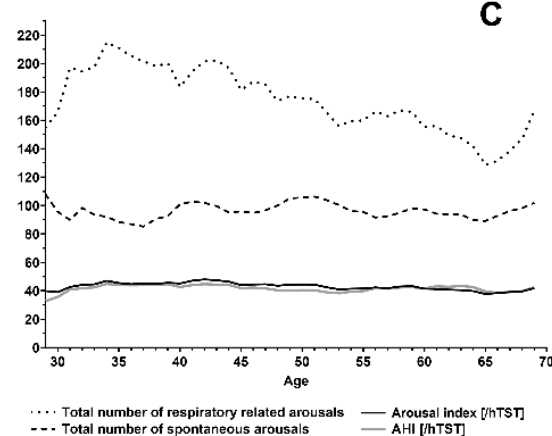

B
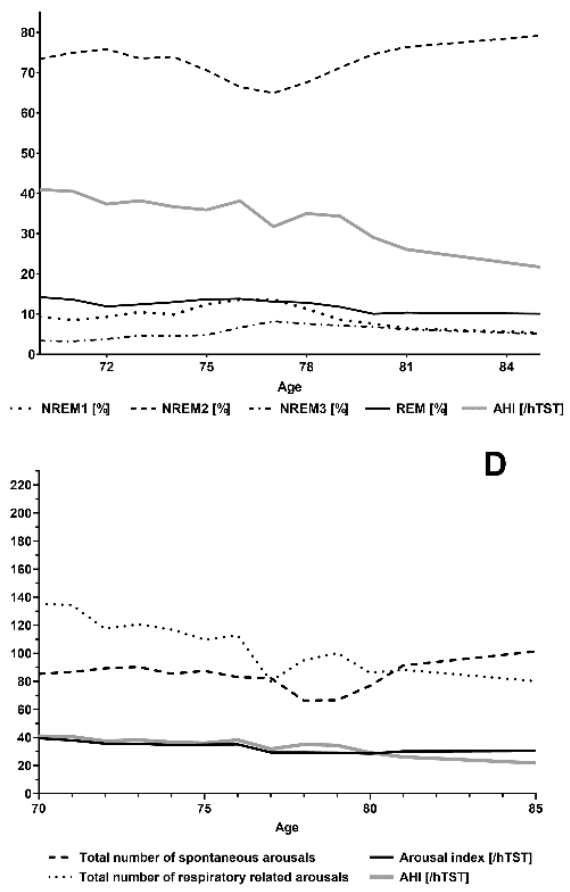

Figure 2. Percentual proportion of sleep cycles in controls (panel A) and seniors (panel B) and sleep fragmentation due to respiratory related arousals (panels $C, D$ ) of patients with OSA. Data are matched for apnea/hypopnea index - AHI. Panels A, B: Patients in the elderly group had lowered proportion of slow-wave NREM3 sleep. ${ }^{*} \mathrm{p}=0,005$ NREM3\%, controls vs. seniors. Panels C, D: In younger people with OSA, arousal index on diagnostic polysomnography night almost corresponded with their apnea/hypopnea index. With advancing age, the number of spontaneous arousals has an increasing tendency and adds to overall sleep fragmentation of seniors with OSA.

A

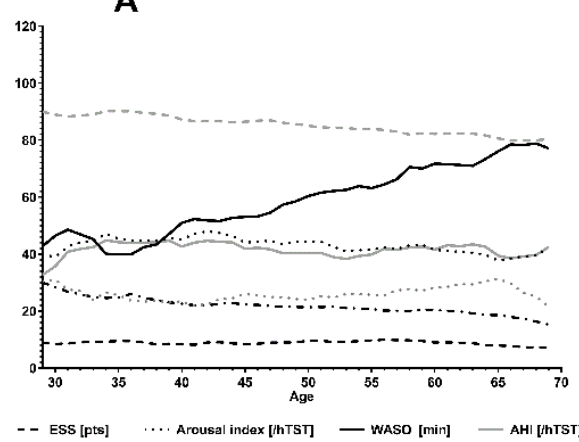

-- ESS [pts] … Arousal index [hTST] - WASO [min] - AHI [hTST]
B

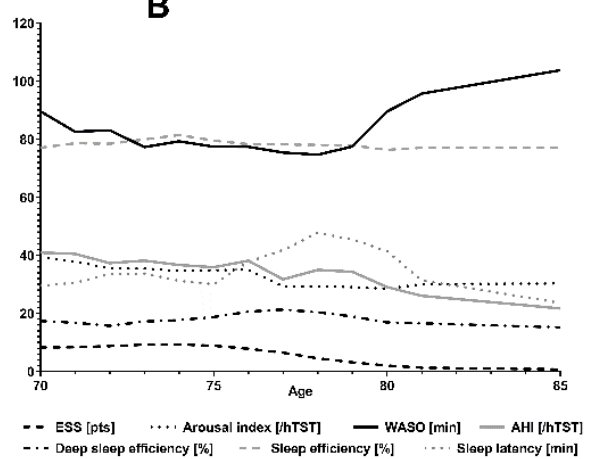

Figure 3. Sleepiness and sleep efficiency in patients with OSA. Subjective perception of sleepiness defined by ESS starts to decrease in older people with OSA, as the AHI and respiratory related arousals decrease, despite the sleep latency and WASO periods are prolonged and deep sleep efficiency is below $80 \%$. ESS - Epworth sleepiness score pre-polysomnography, WASO - wake after sleep onset, /hTST - events per hour of total sleep time.

The number of spontaneous arousals partially adds to the final picture of sleep fragmentation, taking into consideration that seniors with median age 73 years had lower respiratory related arousal index components resulting in significantly lower total arousal indexes while experiencing similar numbers of spontaneous arousals during the night. With increasing age, there was no rapid increase in AHI or respiratory related arousals 
and we saw a rise in the number of spontaneous arousals. (Figure 2, panels C, D). The elders had prolonged latency of sleep onset and significantly longer wake periods after sleep onset (WASO). They had shorter duration of slow-wave NREM3 sleep and conformingly, lower efficiency of deep sleep and lower efficiency of sleep in general (Table 5, Figure 3).

Total sleep time in people over 70 years of age was notably shorter $(315,96 \pm 7,039 \mathrm{~min}$. vs. 348,46 $\pm 1,386$ min., $p=0,035, M E A N \pm S E$, seniors vs. controls) (Table 5). The majority of subjects $(64,706 \%)$ preferred nasal masks over oronasal masks $(35,294 \%)$. The nasal mask was considered more comfortable and less limiting. Majority of patients preferred a nasal mask for personal comfort and because they were able to call for help or assistance when in need during the nighttime.

Pressure support settings in the CPAP treatment of elderly patients were significantly lower $(6,5 \pm 2$ vs. $8,59 \pm 3 \mathrm{cmH} 2 \mathrm{O}, \mathrm{p}<0,001, \mathrm{MED} \pm \mathrm{IQR}$, seniors vs. controls) as partially predicted by lower AHI values in the elderly group. Compliance hours were slightly suboptimal in the elderly group, achieving 3,991 $\pm 3,804$ hours/day with bigger variation in between the subjects versus 5,547 $\pm 3,465$ hours/day for the controls, (MED $\pm I Q R, p<0,001)$.

Spearman correlation coefficient between age in the senior group and their daily compliance and age vs. AHI respectively reached negative values $(r=-0,05014$ and $r=-$ $0,1869, \mathrm{CI} 95 \%, \mathrm{p}<0,0001)$. Sleep fragmentation in elderly was promoted also to suboptimal CPAP adherence, as the probability of CPAP use $\geq 4$ hours per day was less than $20 \%$ in patients over 70 years. Further, despite naturally occurring sleep fragmentation worsened by OSA, less than $30 \%$ of elderly patients would score $\geq 10$ points on Epworth sleepiness scale. However, in people diagnosed with OSA between 20-40 years old, we demonstrated over $90 \%$ probability of scoring excessive daily sleepiness on Epworth sleepiness scale (ESS $\geq 10$ points pre-PSG) in line also with over $90 \%$ probability of optimal CPAP daily use ( $\geq 4$ hours per day) (Figure 4 ).

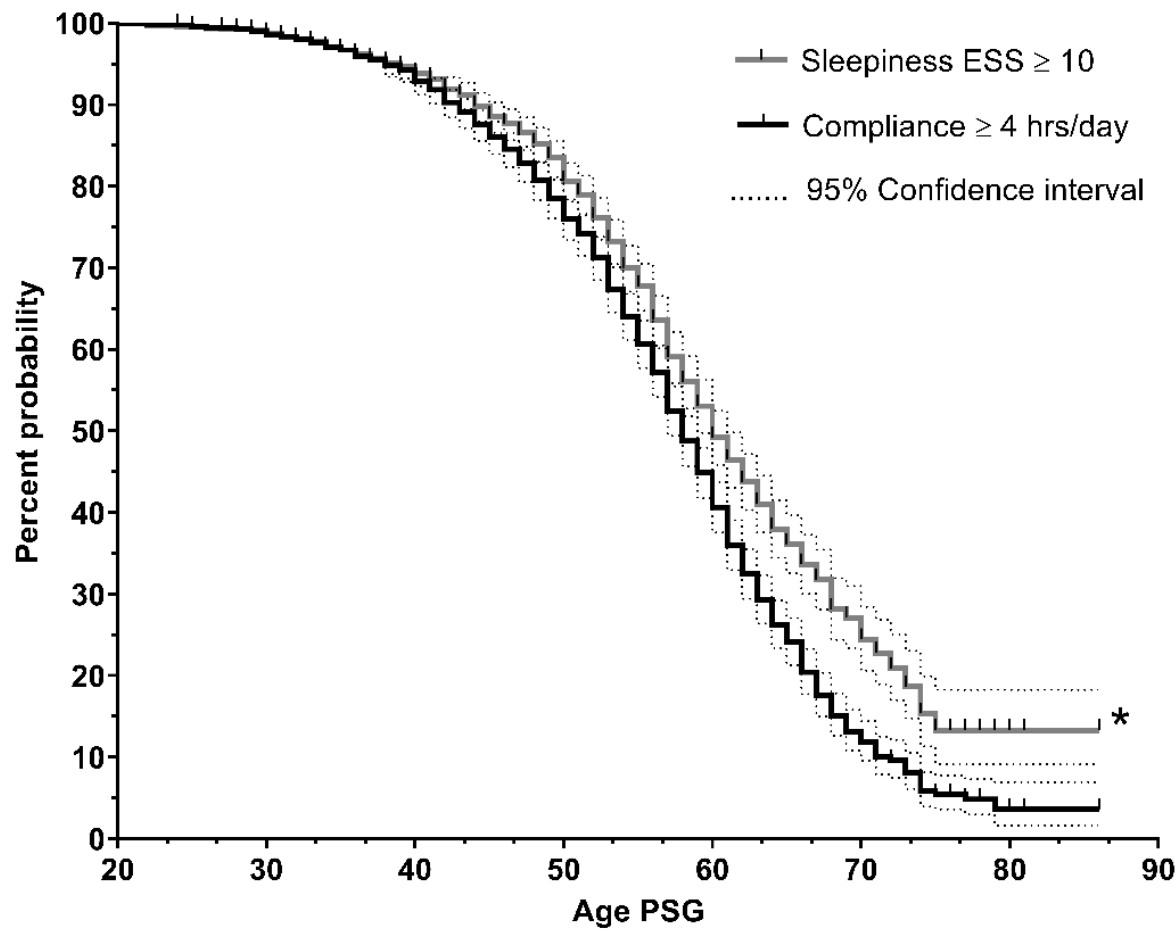

Figure 4. Perception of daily sleepiness of patients with OSA versus CPAP compliance. The probability to indicate excessive daily sleepiness based on Epworth sleepiness score is higher than the probability that the older people stay above 4 hours of daily CPAP use, $\left({ }^{*} p<0,0001\right)$. The data clearly shows that with advancing age the probability of elderly people who are able conform to the compliance criteria of 4 hours CPAP use per day decreases. Age PSG - age at the time of OSA diagnosis and initiation of CPAP therapy, ESS - Epworth sleepiness score at the time of OSA diagnosis. 
Table 5. As supplementum. Polysomnography findings. Seniors versus controls, CI 95\%. BMI - Body mass index [kg/m2], ESS - Epworth sleepiness score [0-24 min-max], SL - Sleep latency [min], NREM1 - Total duration of non-rapid eye movement sleep stage 1 [min], NREM2 - Total duration of non-rapid eye movement sleep stage 2 [min], NREM3 - Total duration of non-rapid eye movement sleep stage 3 [min], NREM1\% - Proportion of NREM 1 [\%], NREM2\% - Proportion of NREM 2 [\%], NREM3\% - Proportion of NREM 3 [\%], REM - Total duration of rapid eye movement sleep [min], REM\% - Proportion of REM [\%], RC - Number of REM cycles, RL - REM latency [min], SPT - Sleep period time [min], TST - Total sleep time [min], SPT/TST - Sleep efficiency, DSE - Deep sleep efficiency ((REM+NREM3)/TST), WASO - Wake after sleep onset [min], ArIndx - Arousal index [arousals per hour of TST], sArousals - Total number of spontaneous arousals, rArousals - Total number of respiratory related arousals, AHI - Apnea/hypopnea index, RDI REM - Respiratory disturbance index in REM sleep [per hour of REM], RDI NREM - Respiratory disturbance index in NREM sleep [per hour of NREM], DesREM - Desaturation index during REM [duration/hour], DesNREM - Desaturation index during NREM [duration/hour].

\section{Discussion}

\subsection{Optimal sleep duration}

Many international sleep and health organizations have their own sleep duration recommendations and there is only a limited amount of data on optimal sleep duration in the elderly population. The United States National Foundation recommends average sleep duration 7 to 9 hours for young adults and adults, and 7-8 hours of sleep is recommended for older adults [8]. CPAP compliance and adherence criteria should allow space for individual sleep pattern variability and take into consideration physiological age-related changes in sleep duration. Our results partially suggest an answer to the USA National Sleep Foundation question on the ratio of daily sleepiness caused due to sleep disordered breathing in older people with OSA [8]. We have found that in groups matched for AHI and ESS, seniors had considerably lower respiratory related arousal index components, while sustaining a similar number of spontaneous arousals and longer periods of wakefulness after sleep onset during the night (Table 5). With advancing age, the number of respiratory related arousals started to decrease, and the proportion of spontaneous arousals increased (Figure 2, panels C, D).

\subsection{Polysomnography - REM sleep}

Detailed role of REM sleep is not fully understood and elucidated until today. The general concept of sleep fragmentation and lowered sleep efficiency is a well-established fact in geriatric medicine. In 2007, Floyd extensively reviewed changes in REM sleep percentage over the adult lifespan in 382 English-language research reports but did not include the number of REM cycles as a variable, nor did not specifically study OSA patients [15]. From a neurological point of view, our findings on the REM sleep in elderly population with OSA showed one notable trait. The onset of first REM sleep cycle and the percentual proportion of REM sleep during the night seems to be preserved also in older people over 70 years of age, even when other representative factors of sleep quality, efficiency and quantity are already disrupted. Remarkably, the latter remains valid even while the number of REM cycles starts to decrease (Table 5).

\subsection{Comorbid insomnia}

When discussing relevant age-related OSA comorbidities, we must not omit other sleep disturbances. Results of this observational study support the recently published concept of so-called COMISA syndrome - comorbid insomnia and OSA [16], particularly in the elderly population. While finding similar AHI, RDI and desaturation indexes in both groups (Table 5), the data on sleep quality and quantity and CPAP daily use suggest possible high prevalence of comorbid OSA and insomnia specifically in the elderly population. 


\subsection{Upper airway patency}

Interestingly, both groups in our study had similar obstructive AHI, RDI and desaturation indexes, while the elders had significantly lower body-mass index values (Table 5). The findings suggest that a healthy diet and weight control is even more important as we age. Overweight and obesity, together with increased upper airway patency might be a more serious predicting factor of severity of OSA in elderly patients than in their younger counterparts. Therefore, we must take so-called "non-obese OSA phenotype" into consideration when looking for observable and maybe age-specific trait of OSA patient in our clinics. Unfortunately, there has been only a limited number of studies regarding the impact of aging on the factors contributing to upper airway patency.

\subsection{CPAP adherence}

It was a positive finding, that the majority of patients over seventy years of age, who were tested positive for obstructive sleep apnea/hypopnoea syndrome, tolerate the continuous positive airway pressure therapy, they are motivated to use the ventilatory device and should not be omitted from benefiting from effective CPAP therapy. Only in a minimal number of the subjects was the CPAP treatment not positively accepted, despite being diagnosed with moderate to severe OSA. In compliant elderly people, nasal mask was significantly preferred over oronasal mask - adding some factor of personal comfort and freedom in the therapy. A "smaller" mask was described as less limiting, allowing the patient to call immediate help or assistance when needed.

The outcomes of this study in patients over 70 years suggest that the age is a relevant factor for evaluation of adequate CPAP therapy adherence (Figure 5). Patients' daily compliance is considered insufficient when they fail to reach 4 hours of device use per day. The length of daily CPAP therapy for a minimum of 4 hours is a golden standard for effective management of OSA. Roots of such a criterion probably lie in the recommended 8 hours of sleep per day, out of which at least half should be under effective CPAP ventilation in patients with OSA. Our results show that elderly people struggle to comply with this specific criterion and after the age of 70 years, the probability of optimal compliance declined to less than $20 \%$. Hence, we hypothesize that the factor of age should be incorporated into the tools for the assessment of therapy quality, compliance and effectiveness.

\subsection{Limitations}

There are several limitations of the study. Seniors often consider their lowered sleep quality to be a part and a consequence of physiological aging and do not seek an expert consultation or tend to misinterpret their problems. Further studies emerging in this topic must take into account several specific aspects of aging and find a definition for healthy sleep in elderly population. Another limiting factor not to be excluded is the first night effect, which undoubtedly influenced polysomnography data in both groups and is frequently present in most of the polysomnography studies. The performing personnel must be trained to explain the procedure and importance of the sleep study so the patient feels as comfortable as possible, and the investigating personnel must be aware of this ofteninseparable feature.

One of the most considerable downsides of a retrospective observational study is a limited or non-existent comparison group of healthy subjects, or in our case, a low number of subjects not suffering from obstructive sleep apnea to compare. Those were excluded from medical investigation based on respiratory polygraphy screening. Therefore, sleep parameters were evaluated and compared to normative adult reference values as in Table 3.

\section{Conclusions}

We want to underline the importance of design and validation of age-specific diagnostic tools to assess daily sleepiness and maybe also OSA severity in the elderly. The 
results also address a need for adjustment of commonly used sleepiness scales and questionnaires for the senior population. CPAP compliance and adherence criteria could be adapted to age, so that we are able to provide optimized and best-tailored treatment for our patient.

Author Contributions: For research articles with several authors, a short paragraph specifying their individual contributions must be provided. The following statements should be used "Conceptualization, S.G. and V.B.; methodology, S.G. and V.B.; validation, S.G. and V.B.; formal analysis, S.G.; investigation, S.G.; resources, S.G.; data curation, S.G.; writing-original draft preparation, S.G.; writing - review and editing, S.G.; visualization, S.G.; supervision, V.B.; project administration, S.G. and V.B. All authors have read and agreed to the published version of the manuscript."

Funding: “This research received no external funding"

Data Availability Statement: Data is available on request from the corresponding author.

\section{Acknowledgments: None}

Conflicts of Interest: "The authors declare no conflict of interest." "No funders had any role in the design of the study; in the collection, analyses, or interpretation of data; in the writing of the manuscript, or in the decision to publish the results".

\section{References}

[1] McMillan A, Morrell MJ. Sleep disordered breathing at the extremes of age: the elderly. Breathe (Sheff). 2016;12(1):50-60. doi:10.1183/20734735.003216

[2] Ancoli-Israel S, Gehrman P, Kripke DF, Stepnowsky C, Mason W, Cohen-Zion M, Marler M. Long-term follow-up of sleep disordered breathing in older adults. Sleep Med. 2001 Nov;2(6):511-6.

[3] Morrell MJ, Finn L, McMillan A, Peppard PE. The impact of ageing and sex on the association between sleepiness and sleep disordered breathing. Eur Respir J. 2012 Aug;40(2):386-93.

[4] Boselli M, Parrino L, Smerieri A, Terzano MG. Effect of age on EEG arousals in normal sleep. Sleep. 1998 Jun 15;21(4):351-7.

[5] Posadas, T., Oscullo, G., Zaldívar, E., Garcia-Ortega, A., Gómez-Olivas, J. D., Monteagudo, M., \& Martínez-García, M. A. (2020). Treatment with CPAP in Elderly Patients with Obstructive Sleep Apnoea. Journal of clinical medicine, 9(2), 546. https://doi.org/10.3390/jcm9020546

[6] Martínez-García MÁ, Chiner E, Hernández L, Cortes JP, Catalán P, Ponce S, Diaz JR, Pastor E, Vigil L, Carmona C, Montserrat JM, Aizpuru F, Lloberes P, Mayos M, Selma MJ, Cifuentes JF, Muñoz A; Spanish Sleep Network. Obstructive sleep apnoea in the elderly: role of continuous positive airway pressure treatment. Eur Respir J. 2015 Jul;46(1):142-51. doi: 10.1183/09031936.00064214.

[7] Netzer NC Chair, Ancoli-Israel S Co-Chair, Bliwise DL, Fulda S, Roffe C, Almeida F, Onen H, Onen F, Raschke F, Martinez Garcia MA, Frohnhofen H. Principles of practice parameters for the treatment of sleep disordered breathing in the elderly and frail elderly: the consensus of the International Geriatric Sleep Medicine Task Force. Eur Respir J. 2016;48(4):992-1018. doi: 10.1183/13993003.01975-2015

[8] Hirshkowitz M, Whiton K, Albert SM, Alessi C, Bruni O, DonCarlos L, Hazen N, Herman J, Adams Hillard PJ, Katz ES, Kheirandish-Gozal L, Neubauer DN, O'Donnell AE, Ohayon M, Peever J, Rawding R, Sachdeva RC, Setters B, Vitiello MV, Ware JC. National Sleep Foundation's updated sleep duration recommendations: final report. Sleep Health. 2015 Dec;1(4):233-243. doi: 10.1016/j.sleh.2015.10.004.

[9] Gronewold, J., Haensel, R., Kleinschnitz, C., Frohnhofen, H., \& Hermann, D. M. (2019). Sleep-Disordered Breathing in Hospitalized Geriatric Patients with Mild Dementia and Its Association with Cognition, Emotion and Mobility. International journal of environmental research and public health, 16(5), 863. https://doi.org/10.3390/ijerph16050863

[10] Abad VC, Guilleminault C. Sleep and psychiatry. Dialogues Clin Neurosci. 2005;7(4):291-303.

[11] Cooke JR, Ancoli-Israel S. Normal and abnormal sleep in the elderly. Handb Clin Neurol. 2011;98:653-65. doi: 10.1016/B978-0444-52006-7.00041-1.

[12] McCall WV. Sleep in the Elderly: Burden, Diagnosis, and Treatment. Prim Care Companion J Clin Psychiatry. 2004;6(1):9-20.

[13] Onen SH, Onen F. Chronic Medical Conditions and Sleep in the Older Adult. Sleep Med Clin. 2018 Mar;13(1):71-79.

[14] Kryger's Atlas of Clinical Sleep Medicine. Editor(s): Meir H. Kryger, Thomas Roth, William C. Dement, Principles and Practice of Sleep Medicine (Fifth Edition), W.B. Saunders, 2011, Pages e1-e37, ISBN 9781416066453.

[15] Floyd JA, Janisse JJ, Jenuwine ES, Ager JW. Changes in REM-sleep percentage over the adult lifespan. Sleep. 2007;30(7):829-836. doi:10.1093/sleep/30.7.829

[16] Brock MS, Mysliwiec V. Comorbid insomnia and sleep apnea: a prevalent but overlooked disorder. Sleep Breath. 2018 Mar;22(1):1-3. doi: 10.1007/s11325-018-1628-4. 


\section{Table 5. Supplementum: Polysomnography findings}

Seniors ( $\mathrm{n}=162$ ) versus controls ( $\mathrm{n}=448)$, CI 95\%. BMI - Body mass index [kg/m2], ESS - Epworth sleepiness score [0-24 min-max], SL - Sleep latency [min], NREM1 - Total duration of non-rapid eye movement sleep stage 1 [min], NREM2 - Total duration of non-rapid eye movement sleep stage 2 [min], NREM3 - Total duration of non-rapid eye move-ment sleep stage 3 [min], NREM1\% - Proportion of NREM 1 [\%], NREM2\% - Proportion of NREM 2 [\%], NREM3\% - Propor-tion of NREM 3 [\%], REM - Total duration of rapid eye movement sleep [min], REM\% - Proportion of REM [\%], RC - Num-ber of REM cycles, RL - REM latency [min], SPT - Sleep period time [min], TST - Total sleep time [min], SPT/TST - Sleep ef-ficiency, DSE - Deep sleep efficiency ((REM+NREM3)/TST), WASO - Wake after sleep onset [min], ArIndx - Arousal index [arousals per hour of TST], sArousals - Total number of spontaneous arousals, rArousals - Total number of respiratory re-lated arousals, AHI - Apnea/hypopnea index, RDI REM - Respiratory disturbance index in REM sleep [per hour of REM], RDI NREM - Respiratory disturbance index in NREM sleep [per hour of NREM], DesREM - Desaturation index during REM [duration/hour], DesNREM - Desaturation index during NREM [duration/hour]. 


\begin{tabular}{|c|c|c|c|c|c|c|c|c|c|c|c|c|c|c|}
\hline Controls & Age & BMI & ESS & SL & NREM1 & NREM1\% & NREM2 & NREM2\% & NREM3 & NREM3\% & REM & REM\% & RC & RL \\
\hline Mean & 52,019262 & 33,493234 & 8,9811563 & 26,310881 & 29,505165 & 9,231949282 & 244,1888 & 69,976354 & 21,556287 & 6,1364533 & 52,171174 & 14,579251 & 2,809524 & 134,537 \\
\hline Standard & 0,2099792 & 0,1388807 & 0,1199577 & 0,5750577 & 0,5483307 & 0,19141077 & 1,416685 & 0,2875151 & 0,5568488 & 0,1543177 & 0,6537498 & 0,1682141 & 0,031831 & 1,89351 \\
\hline Median & 54 & 32,4622 & 9 & 18,5 & 22 & 6,550802 & 249 & 70,36505 & 13 & 3,7753495 & 51 & 14,66459 & 3 & 112,5 \\
\hline Maximum & 69 & 75,11338 & 24 & 400 & 342 & 93,02885 & 439,5 & 99,60265 & 387,5 & 97,85354 & 192,5 & 51,83616 & 12 & 438,5 \\
\hline Minimum & 19 & 15,57093 & 0 & 0 & 1 & 0 & 0 & 0 & 0 & 0 & 0 & 0 & 0 & 0 \\
\hline Interquarti & 14 & 8,699145 & 8 & 23 & 22,875 & 7,669524 & 89,5 & 16,917752 & 35 & 9,9982157 & 46 & 11,599079 & 2 & 113,5 \\
\hline Seniors & Age & BMI & ESS & SL & NREM1 & NREM1\% & NREM2 & NREM2\% & NREM3 & NREM3\% & REM & REM\% & $\mathrm{RC}$ & RL \\
\hline Mean & 73,29787 & 32,15182 & 8,238806 & 32,23165 & 27,19928 & 9,907041 & 229,1655 & 72,53434 & 14,91367 & 4,680871 & 43,33237 & 13,32894 & 2,431655 & 131,792 \\
\hline Standard & 0,255188 & 0,524054 & 0,47649 & 3,018827 & 2,015337 & 0,858959 & 6,269558 & 1,071604 & 1,773137 & 0,542774 & 2,662758 & 0,75339 & 0,131975 & 8,80609 \\
\hline Median & 73 & 31,0477 & 8 & 21 & 18,5 & 5,963939 & 237,5 & 73,51525 & 3,5 & 1,308411 & 41 & 13,74408 & 2 & 108,5 \\
\hline Maximum & 87 & 58,82353 & 21 & 201,5 & 148,1 & 63,64347 & 399 & 100 & 114 & 27,70147 & 127,5 & 35,71429 & 8 & 407 \\
\hline Minimum & 70 & 16,43655 & 0 & 0 & 1,5 & 0,482509 & 46,5 & 36,35653 & 0 & 0 & 0 & 0 & 0 & 0 \\
\hline Interquarti & 4 & 7,371865 & 8 & 24 & 19,75 & 8,566219 & 110 & 14,98593 & 27,75 & 8,340286 & 46,75 & 12,86155 & 2 & 136 \\
\hline$p$ value & $<0,0001$ & 0,006 & 0,113 & 0,045 & 0,082 & 0,925 & 0,022 & 0,057 & 0,001 & 0,005 & 0,001 & 0,083 & 0,004 & 0,313 \\
\hline Controls & SPT & TST & SPT/TST & DSE & WASO & sArousals & rArous & ArIndx & AHI & RDI REM & RDI & DesREM & DesNRE & \\
\hline Mean & 409,7292 & 348,4698 & 84,19177 & 21,13622 & 62,694477 & 97,51122 & 163,03 & 41,84823 & 39,83949 & 36,455 & 39,536 & 19,95975 & 17,62618 & \\
\hline Standard & 0,960831 & 1,385663 & 0,277823 & 0,248448 & 1,0236076 & 1,376189 & 3,5189 & 0,518021 & 0,663983 & 0,6568895 & 0,6894574 & 0,406752 & 0,372517 & \\
\hline Median & 416 & 360,6 & 87,73661 & 20,60487 & 50 & 83 & 105 & 36,8 & 33,86139 & 32,800 & 32,400 & 14,7 & 11,7 & \\
\hline Maximum & 567 & 551,7 & 100 & 81,27129 & 337 & 753 & 1179 & 174,8 & 201,1399 & 160,00 & 196,900 & 93 & 94,5 & \\
\hline Minimum & 3 & 9,5 & 0 & 0 & 0 & 0 & 0 & 0 & 0 & 0,000 & 0,000 & 0 & 0 & \\
\hline Interquarti & 43,55 & 82,5 & 15,30303 & 16,98812 & 60,5 & 79 & 222 & 35,8 & 53,27685 & 56,7 & 55,7 & 32,6 & 25,875 & \\
\hline Seniors & SPT & TST & SPT/TST & DSE & WASO & sArousals & rArous & ArIndx & AHI & RDI REM & RDI & DesREM & DesNRE & \\
\hline Mean & 398,29 & 315,9652 & 78,51598 & 18,07546 & 82,42857 & 86,96403 & 116,16 & 35,36331 & 37,13514 & 34,612 & 36,118 & 19,35802 & 15,11353 & \\
\hline Standard & 5,116228 & 7,039181 & 1,333899 & 0,948436 & 4,961927 & 5,03216 & 9,2583 & 1,535349 & 2,239739 & 2,4885326 & 2,3140052 & 1,611275 & 1,321028 & \\
\hline Median & 414,7 & 333 & 82,35294 & 17,64706 & 66,25 & 73 & 79 & 31,4 & 31,75439 & 33,9 & 30,6 & 15,2 & 10 & \\
\hline Maximum & 499 & 546 & 100 & 41,94982 & 248,5 & 313 & 442 & 82,2 & 98,90278 & 102,400 & 101,8 & 80,6 & 69,4 & \\
\hline Minimum & 143 & 86,5 & 27,71998 & 0 & 0 & 0 & 0 & 0 & 0 & 0,000 & 0,000 & 0 & 0 & \\
\hline Interquarti & 55,45 & 108,2 & 21,3493 & 15,49938 & 78,375 & 71 & 148 & 27,7 & 37,70172 & 50,2 & 41,4 & 29,7 & 17,8 & \\
\hline$p$ value & 0,185 & $<0,0001$ & $<0,0001$ & 0,005 & $<0,0001$ & 0,059 & 0,009 & 0,005 & 0,346 & 0,485 & 0,691 & 0,311 & 0,338 & \\
\hline
\end{tabular}

